\title{
Tendências evolutivas dos cirurgiões de catarata presentes no IV Congresso Brasileiro de Catarata e Cirurgia Refrativa
}

\author{
Evolutionary trends of cataract surgeons who attended the IV \\ Brazilian Congress of Cataract and Refractive Surgery
}

Marcony Rodrigues de Santhiago', Beatriz de Abreu Fiuza Gomes², Fernanda Ferreira Pires Gaffree², Vinicius da Silva Varandas ${ }^{3}$, Adroaldo de Alencar Costa Filho ${ }^{4}$

\begin{tabular}{|l|}
\hline$\underline{\text { RESUMO }}$ \\
Objetivo: Dar continuidade ao processo de atualização e aperfeiçoamento da cirurgia \\
de catarata no Brasil, além de obter dados para uma análise comparativa das atuais \\
tendências cirúrgicas nacionais e internacionais. Métodos: Em maio de 2007, foram \\
obtidos dados de 188 participantes de um congresso de Catarata e Cirurgia Refrativa \\
através questionários autoaplicáveis, contendo perguntas objetivas a respeito da cirur- \\
gia de catarata. Análise estatística foi feita através teste Te Qui-quadrado. Resultados: \\
Observou-se que 95,8\% dos participantes praticam a facoemulsificação, enquanto so- \\
mente 4,2\% preferem usar a técnica extracapsular convencional. Para $73,4 \%$ dos par- \\
ticipantes, o bloqueio peribulbar é preferido em relação ao uso de anestésico tópico \\
(20,2\%). A técnica de facoemulsificação mais realizada é a de dividir e conquistar \\
(32,0\%). A hidroximetilcelulose foi o viscoelástico de escolha para $60,8 \%$ dos colabo- \\
radores. O aparelho de facoemulsificação mais usado pelos participantes foi o Infiniti \\
(24,0\%), seguido pelo Legacy $(19,8 \%)$. Conclusão: Os resultados deste estudo foram \\
consistentes com o que era esperado, tendo como base pesquisas semelhantes. Destaca-se \\
um aumento considerável do número cirurgiões adeptos à facoemulsificação e tendênci- \\
as no sentido de maior utilização de incisões corneanas temporais e anestesia tópica.
\end{tabular}

Descritores: Catarata; Extração de catarata/métodos; Extração de catarata/estatística \& dados numéricos; Implante de lente intra-ocular/estatística \& dados numéricos; Tendências; Facoemulsificação; Condutas na prática dos médicos/estatística \& dados numéricos; Procedimentos cirúrgicos refrativos/ estatística \& dados numéricos

\footnotetext{
${ }^{1}$ Estagiário e pesquisador do Setor de Catarata do Hospital das Clínicas da Faculdade de Medicina da Universidade de São Paulo - USP - São Paulo (SP), Brasil;

${ }^{2}$ Residente do $3^{\circ}$ ano do Setor de Oftalmologia da Universidade Federal do Rio de Janeiro - UFRJ - Rio de Janeiro (RJ), Brasil;

'Pós-graduando (Doutorado) da Universidade Federal do Rio de Janeiro - UFRJ - Rio de Janeiro (RJ), Brasil;

${ }^{4}$ Doutor, Professor Adjunto da Universidade Federal do Rio de Janeiro - UFRJ - Rio de Janeiro (RJ), Brasil;
}

Estudo realizado no Setor de Oftalmologia da Universidade Federal do Rio de Janeiro - UFRJ - Rio de Janeiro (RJ), e no Setor de Oftalmologia da Universidade de São Paulo - USP - São Paulo (SP), Brasil.

Recebido para publicação em: 9/10/2008 - Aceito para publicação em 29/01/2009 


\section{INTRODUÇÃO}

A técnica da facoemulsificação, apresentada inicialmente $^{(1)}$, na década de 60 , revolucionou a facectomia. A rapidez das mudanças neste procedimento cirúrgico, assim como o crescimento vertiginoso do número de praticantes, justifica o empenho em avaliar a situação atual da cirurgia de catarata, suas tendências e as preferências dos cirurgiões ${ }^{(2)}$.

Em vários países são desenvolvidos estudos estatísticos com este propósito ${ }^{(3-6)}$. Nos Estados Unidos (EUA), desde 1984, há pesquisas com os membros da American Society of Cataract and Refractive Surgery (ASCRS), permitindo um melhor conhecimento das preferências e novas tendências da facoemulsificação naquele país ${ }^{(7-11)}$.

No Brasil, o primeiro passo na busca do perfil do cirurgião de catarata foi dado em $1996^{\left({ }^{(12)}\right.}$. No entanto, pesquisas nacionais referentes ao assunto são escassas ${ }^{(12-15)}$. Em 2002, estudo semelhante aos realizados em outros países, ressaltando a importância de uma documentação estatística adequada para o registro das características cirúrgicas nacionais e sua evolução através do tempo, foi realizado ${ }^{(14)}$.

O presente estudo tem como objetivo avaliar o perfil cirúrgico dos participantes do IV Congresso Brasileiro de Catarata e Cirurgia Refrativa (IV CBCR). Além disso, visa obter dados para uma análise comparativa com as tendências nacionais e internacionais, justificando-se por ser o único estudo com este propósito nos últimos 6 anos.

\section{Métodos}

Trata-se de um estudo transversal.

Em 2007, foram distribuídos questionários contendo 20 perguntas simples e objetivas sobre cirurgia de catarata durante o IV Congresso Brasileiro de Catarata e Cirurgia Refrativa (CBCR). A distribuição dos questionários foi aleatória e seu preenchimento foi voluntário e anônimo. A amostragem foi não probabilística. Foram excluídos deste estudo participantes do congresso sem graduação em medicina. Não houve qualquer recompensa financeira para os colaboradores. Perguntas semelhantes às realizadas em estudo prévio foram mantidas para que uma tendência pudesse ser identificada ${ }^{(14)}$. Algumas questões permitiram mais de uma resposta e aquelas incompletas, rasuradas ou nãopadronizadas foram desconsideradas.

Os dados foram transferidos para a planilha Microsoft Excel ${ }^{\varpi}$, sendo a seguir analisados estatistica- mente usando o método Qui-Quadrado para avaliar a relação entre variáveis estudadas por este trabalho e teste $\mathrm{T}$ foi realizado para comparação de uma mesma proporção de amostras diferentes, com intervalo de confiança de $95 \%$.

\section{Resultados}

Foram obtidos dados de 188 participantes, 10,6\% dos participantes da pesquisa estavam cursando a residência médica, sendo que a maioria destes $(n=12)$ cursava o último ano e não havia nenhum residente do primeiro ano. A maior parte (53,1\%) tinha até 10 anos de formado.

Ao analisarmos a subespecialidade praticada, observou-se grande diversidade, com predomínio do segmento anterior, sendo que "catarata" representou $55,4 \%$.

Quanto ao volume cirúrgico, verificou-se que $57,1 \%$ dos participantes realizam até 20 cirurgias/mês, enquanto que $17,2 \%$ fazem mais de 30 cirurgias por mês.

Dentre os pesquisados, $4,2 \%$ praticam, como rotina, a facectomia extracapsular convencional, $95,8 \%$ a facoemulsificação, sendo que destes últimos, 3,3\% praticam a técnica de microincisão coaxial.

O bloqueio peribulbar é a anestesia preferencialmente utilizada pelos entrevistados deste estudo $(73,4 \%)$. Anestesia tópica com colírio representou 19,7\% das respostas e anestesia tópica intracamerular 1,6\%.

Relacionando o volume cirúrgico com o tipo de anestesia utilizado, apurou-se que $78,9 \%$ daqueles que realizam preferencialmente anestesia tópica, apresentam um volume cirúrgico maior que 20 cirurgias/mês e o mesmo percentual utilizam uma incisão de $2,75 \mathrm{~mm}$ ou menor. De fato, a avaliação através do teste qui-quadrado evidenciou uma inter-relação entre o tipo de anestesia e o volume cirúrgico. Por outro lado, não houve relação entre o tamanho da incisão e o tipo de anestesia, para um nível de significância de 5\%.

Em relação à incisão cirúrgica, esta é realizada preferencialmente na esclera por 5,8\% dos participantes, $21,3 \%$ no limbo e $72,9 \%$ em córnea clara. Para sua realização, 73,4\% dos respondentes usam lancetas descartáveis. O sítio preferencial da incisão é temporal para 35,6\% dos oftalmologistas, frontal (às 12 horas) para $29,3 \%$, de acordo com lado operado para $27,7 \%$ e, baseando-se em dados de topografia corneana (eixo mais curvo) para 7,4\%.

Ainda em relação à incisão, o tamanho utilizado por $45,2 \%$ dos participantes é de $2,75 \mathrm{~mm}$. Menor que $2,75 \mathrm{~mm}$ foi referido por $9,7 \%$ e maior que $3 \mathrm{~mm}$ por $37,6 \%$ dos participantes. 
Ao analisarmos a substância viscoelástica, verificou-se que a hidroximetilcelulose é a mais utilizada $(60,8 \%)$. Por outro lado, o aparelho de facoemulsificação mais usado pelos participantes desta pesquisa é o Infiniti ${ }^{\circledR}$ $(24,0 \%)$, seguido pelo Legacy ${ }^{\circledR}(19,8 \%)$ e Universal ${ }^{\circledR}$ $(17,7 \%)$.

Dentre os cirurgiões que utilizam o aparelho de facoemulsificação moderno (Infiniti ${ }^{\circledR}$ ), notou-se que $30,0 \%$ usam metilcelulose enquanto que $60,9 \%$ usam Viscoat $^{\circledR}$ ou Duovisc ${ }^{\circledR}$.

As lentes intraoculares (LIOs) mais utilizadas são as acrílicas hidrofóbicas (43,3\%), seguidas pelas lentes de polimetilmetacrilato $(29,7 \%)$. As LIOs multifocais são as mais usadas por apenas 3,0\% dos participantes. No caso de LIOs dobráveis, a maioria $(79,1 \%)$ faz uso de injetores.

Constatou-se que a maior parte $(60,4 \%)$ não utiliza nenhuma substância no frasco da solução salina balanceada e que 32,5\% dos participantes não adotam medidas específicas para evitar a opacificação de capsular posterior.

Em relação a técnica de fratura do núcleo na cirurgia de facoemulsificação, 32,0\% adotam a técnica de "dividir e conquistar", 25,8\% "phaco-chop", 27,8\% "stop and chop"e 14,4\% "quick-chop".

No que se refere à refração pós-operatória, 53,2\% dos entrevistados planejam obter emetropia, enquanto que para $38,3 \%$ o objetivo é a miopia.

Diante de um paciente com catarata e glaucoma com indicação cirúrgica, a maioria dos oftalmologistas $(61,1 \%)$ prefere indicar a cirurgia combinada. Destes, $72 \%$ não usam incisão combinada, enquanto $28 \%$ utilizam incisão combinada.

Por último, o antibiótico usado no pós-operatório para a maioria $(83,1 \%)$ são as quinolonas de quarta geração, sendo que gatifloxacina e moxifloxacina apresentam percentuais semelhantes.

\section{Discussão}

Com o intuito de traçar o perfil cirúrgico dos participantes do IV CBCR e de identificar as tendências referentes à cirurgia de catarata, foi feita esta pesquisa. No entanto, como a amostragem deste estudo, assim como a de outros estudos de linha de pesquisa similar ${ }^{(14)}$, foi baseada em participantes de um congresso, a interpretação dos resultados exige cautela ${ }^{(2)}$. Supõe-se que a maioria dos participantes de congresso é representada por aqueles que priorizam o aperfeiçoamento técnicocirúrgico em catarata e refrativa, sendo este fato considerado um viés caso os resultados fossem extrapolados

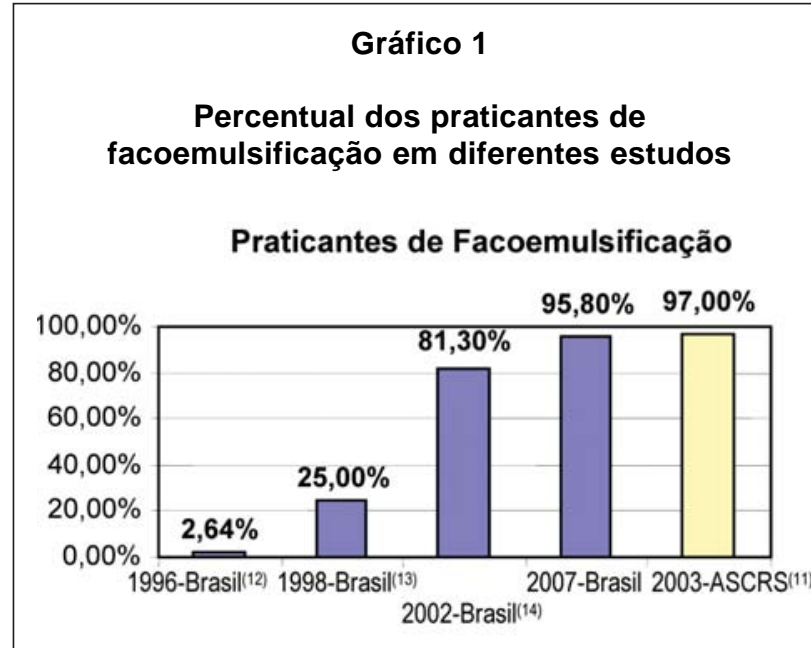

Os números entre parênteses correspondem à referência bibliográfica da fonte. Este artigo foi identificado como 2007-Brasil

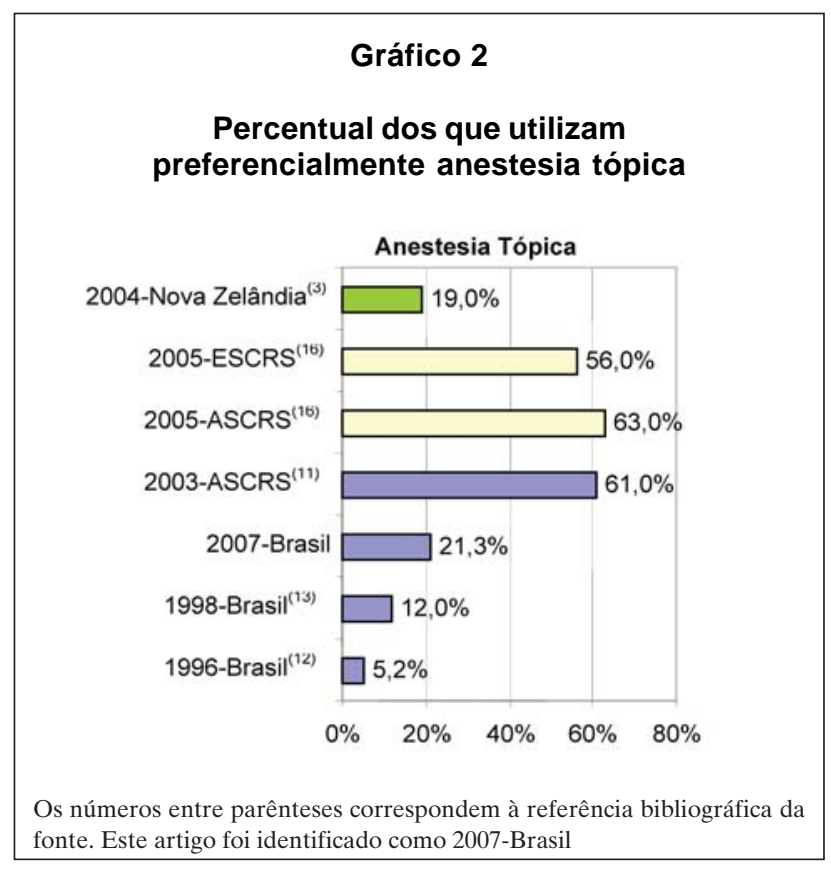

para realidade brasileira, além é claro, do número de entrevistados não representar de maneira significativa o total de cirurgiões no país.

Dentre os mais relevantes achados deste estudo, encontra-se o alto percentual de praticantes de facoemulsificação (Gráfico 1). Em estudo realizado há cerca de 10 anos, no Brasil, somente 2,64\% ${ }^{(12)}$ faziam facoemulsificação de rotina comparado com os atuais 95,8\% verificados durante o IV CBCR, evidenciando um aumento significativo $(\mathrm{p}<0.005)$. Na avaliação norte realizada entre os membros da ASCRS em $2003^{(10)}, 97 \%$ dos oftalmologistas praticavam facoemulsificação 
(percentual mantido desde 2000) ${ }^{(8-11)}$. Através do teste T, observamos ausência de diferença para nível de significância de $5 \%$ entre o percentual atual de facoemulsificação encontrado em nosso estudo e o obtido na avaliação dos membros da ASCRS em 2003.

Em relação à anestesia, observou-se que 74,7\% dos participantes que realizam facoemulsificação utilizam, de rotina, o bloqueio peribulbar, que também é o preferido entre os cirurgiões de extracapsular. No entanto, nota-se uma tendência ao aumento do uso de anestesia tópica. Em 1996, apenas 5,2\% faziam uso da mesma ${ }^{(12)}$. Já em 1998, há relatos de cerca de $12 \% \cdot{ }^{(13)} \mathrm{Em}$ 2002, 38,1\% utilizavam anestesia tópica eventualmente e $8,3 \%$ em $100 \%$ dos $\operatorname{casos}^{\left({ }^{(14)}\right.}$ (Gráfico 2).

Outra evidência do aperfeiçoamento técnico-cirúrgico seria a preferência pelas incisões corneanas, as quais são realizadas por $72,9 \%$ dos entrevistados. Em relação a 2002, notou-se aumento e a mesma tendência tem sido observada em estudos realizados em outros países ${ }^{(3,1,1,4,16)}$. Sabe-se que as incisões em córnea clara apresentam inúmeras vantagens sobre as incisões de túnel escleral, destacando-se, por exemplo, a relativa neutralidade do astigmatismo ${ }^{(17)}$.

Existe uma tendência, entre os entrevistados, de utilização de incisões temporais. A transição para preferência deste local de incisão sobre a posição das 12 horas ocorreu em 1998 entre os membros da sociedade americana e em 2001 da sociedade européia de catarata e refrativa (ESCRS) ${ }^{(16)}$. Verificou-se neste estudo que, $35,6 \%$ realizam incisão temporal comparados com $29,3 \%$ referentes à incisão às 12 horas. $7,4 \%$ dos participantes realizam no eixo mais curvo de acordo com a topografia corneana. Esta tendência de usar incisão temporal parece aumentar com o volume cirúrgico ${ }^{(11)}$.

Dentre os participantes que utilizam uma incisão menor que $2,75 \mathrm{~mm}, 100 \%$ apresentam volume cirúrgico maior que 30 cirurgias/mês e $77,7 \%$ utilizam ou o $\operatorname{Legacy}^{\oplus}(11 \%)$ ou o Infiniti $(66,7 \%)$ como aparelhos de facoemulsificação, mostrando que parece haver influência tanto da experiência do cirurgião quanto da tecnologia disponível na escolha do tipo de incisão $(\mathrm{p}<0.005)$.

Em relação ao material viscoelástico, a tendência observada durante o IV CBCR foi compatível com o estudo de $20022^{(14)}$, mostrando a maior utilização da metilcelulose. Tal fato pode não significar a preferência dos cirurgiões entrevistados, mas sim a maior disponibilidade, uma vez que, entre os cirurgiões que utilizam o aparelho de facoemulsificação Infiniti ${ }^{\oplus}$, notou-se que Viscoat $^{\oplus}$ ou Duovisc ${ }^{\circledast}$ são os mais utilizados.

Quanto ao aparelho de facoemulsificação mais

\section{Gráfico 3}

\section{Preferência dos entrevistados em relação à técnica de fratura do núcleo: trabalho atual e estudo realizado em $2002^{(14)}$}

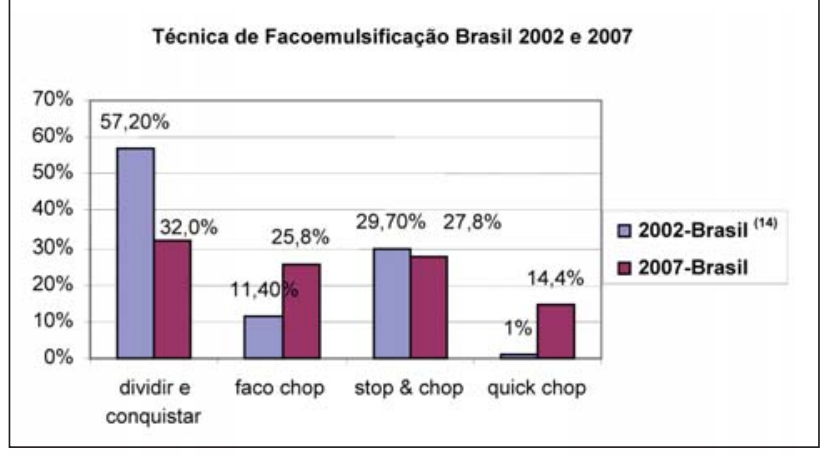

utilizado, observou-se importante diversidade. Porém, o maior percentual isolado $(24,0 \%)$ obtido foi o relacionado ao uso do Infiniti ${ }^{\oplus}$ o que pode ser justificado pelo número de subespecialistas em catarata presentes no estudo (91\% dos subespecialistas em catarata operam no Infiniti ${ }^{\oplus}$.

Lentes intraoculares multifocais são usadas apenas por 3\% dos entrevistados, o que pode refletir a necessidade de maior aprofundamento nesta tecnologia entre os participantes do Congresso.

De acordo com os pesquisados neste estudo, parece haver um declínio entre os praticantes da técnica "dividir e conquistar" em favor das técnicas de núcleo fratura $(\mathrm{p}<0.005)$, apesar de ainda ser a técnica predominante nesta análise.

Em se tratando de antibioticoterapia pós-operatória de escolha, observou-se perfil semelhante ao dos membros da ASCRS, com resultados semelhantes entre moxifloxacino e gatifloxacino. Curiosamente, dados de 2005 mostram que, entre os membros da ESCRS, a tobramicina foi a preferida ${ }^{(16)}$, sendo no presente estudo minoria.

\section{Conclusão}

Os resultados deste estudo foram consistentes com o que era esperado, tendo como base pesquisas semelhantes e foi possível análise comparativa com estudo anterior (Gráfico 3). Destaca-se um aumento considerável do numero de cirurgiões adeptos à facoemulsificação e tendências no sentido de maior utilização de incisões corneanas temporais e anestesia tópica. 


\section{Abstract}

Purpose: To continue the updating and improving process of the cataract surgery in Brazil, through recently pulled out and documented registries. In addition, we want to obtain data for a comparative analysis of the current national and international surgical trends. Methods: In May 2007, data were obtained from 188 participants of a Congress of Cataract and Refrative Surgery who answered simple and multiple-response questions about cataract surgery. Data were analyzed by statistic means of Chisquare test and T test. Results: $95.8 \%$ of respondents practice phacoemulsification, while only $4.2 \%$ perform the conventional planned extracapsular cataract extraction. For $73.4 \%$ of respondents, the periocular block is preferred instead of topical anesthesia $(20.2 \%)$. The most practiced phacoemulsification technique is the 4section divide and conquer $(32.0 \%)$. The hidroximetilcelulosis is the ophthalmic viscosurgical device chosen by $60.8 \%$ of respondents. The most used phaco machine is Infiniti ${ }^{\circledR}(24.0 \%)$, followed by Legacy ${ }^{\circledR}$ (19.8\%). Conclusion:According to this paper is possible to suggest a tendency to high the surgeons who practice clear corneal incision and topical anesthesia. Besides that it was possible to suggest that in ten years the phacoemulsification seems to be consolidated as the surgeons's procedure of choice.

Keywords: Cataract; Cataract extraction/methods; Cataract extraction/statistics \& numerical data; Lens implantation, intraocular/statistics \& numerical data; Trends; Phacoemulsification; Physician's practice patterns/statistics \& numerical data; Refractive surgical procedures

\section{RefêrenCIAS}

1. Kelman CD. Phaco-emulsification and aspiration. A new technique of cataract removal. A preliminary report. Am J Ophthalmol. 1967;64(1):23-35.

2. Kohnen $\mathrm{T}$. The value of surveys for cataract and refractive surgery. J Cataract Refract Surg. 2003;29(9):1647-8.
3. Elder M, Leaming D, Hoy B. New Zealand cataract and refractive surgery survey 2004. Clin Experiment Ophthalmol. 2006;34(5):401-10.

4. Chaidaroon W, Tungpakorn N, Puranitee P. Current trends in cataract surgery in Thailand - 2004 survey. J Med Assoc Thai. 2005;88 Suppl 9:S43-50.

5. Zemba M, Filip M, Bobeico V, Andrei S, Anton O. [Cataract surgery in Romania - 2003]. Oftalmologia. 2004;48(4):64-9. Romanian.

6. Oshika T, Amano S, Araie M, Majima Y, Leaming DV. Current trends in cataract and refractive surgery in Japan: 1999 survey. Jpn J Ophthalmol. 2001;45(4):383-7.

7. Leaming DV. Practice styles and preferences of ASCRS members - 1999 survey. J Cataract Refract Surg. 2000;26(6):913-21.

8. Leaming DV. Practice styles and preferences of ASCRS members - 2000 survey. American Society of Cataract and Refractive Surgery. J Cataract Refract Surg. 2001;27(6):948-55.

9. Leaming DV. Practice styles and preferences of ASCRS members - 2001 survey. J Cataract Refract Surg. 2002;28(9):1681-8.

10. Leaming DV. Practice styles and preferences of ASCRS members - 2002 survey. J Cataract Refract Surg. 2003;29(7):1412-20.

11. Leaming DV. Practice styles and preferences of ASCRS members - 2003 survey. J Cataract Refract Surg. 2004;30(4):892-900.

12. Siqueira JAM, Trindade FC. O perfil do cirurgião de catarata no Brasil -1996. Rev Bras Oftalmol. 1996;55(11):855-60.

13. Resende F. Perfil do cirurgião de catarata no Brasil. Oftalmol Foco. 1998; 57:21.

14. Vieira Netto M, Noleto S, Komatsu F, Guedes J, Centurion V, Figueiredo CG, Magalhães R. Tendências atuais da cirurgia de catarata no Brasil - 2002. Rev Bras Oftalmol. 2002;61(12): 856-61.

15. Loeff RF, Corrêa ZMS, Marcon AS, Freitas AM, Marcon IM. O perfil dos cirurgiões de catarata no sul do Brasil. Arq Bras Ofttalmol. 2003;66(5):621-5

16. Leaming DV. Snapshots. J Cataract Refract Surg. 2006;32(9):1401-2.

17. Rezende F. Cirurgia de catarata. 2a ed. Rio de Janeiro: Cultura Médica; 2002.
ENDEREÇO PARA CORRESPONDÊNCIA:
Rua Teodoro Sampaio ${ }^{\circ} 498$ - apto. 35 - Pinheiros
CEP 05406-000 - São Paulo - SP
E mail: marconysanthiago@hotmail.com 